\title{
Article \\ Know Your Safe Drinking Skills: Adaptation Strategies for the College Effect
}

\author{
Carolyn A. Lin * , John L. Christensen and Anne Borsai Basaran
}

Citation: Lin, Carolyn A., John L. Christensen, and Anne Borsai Basaran. 2022. Know Your Safe Drinking Skills: Adaptation Strategies for the College Effect. Social Sciences 11: 18. https:// doi.org/10.3390/socsci11010018

Academic Editor: Timo Juhani Lajunen

Received: 16 October 2021 Accepted: 3 January 2022 Published: 8 January 2022

Publisher's Note: MDPI stays neutral with regard to jurisdictional claims in published maps and institutional affiliations.

Copyright: (C) 2022 by the authors. Licensee MDPI, Basel, Switzerland. This article is an open access article distributed under the terms and conditions of the Creative Commons Attribution (CC BY) license (https:// creativecommons.org/licenses/by/ $4.0 /)$.

\author{
Department of Communication, University of Connecticut, Storrs, Mansfield, CT 06269-1295, USA; \\ john.christensen@uconn.edu (J.L.C.); anne.borsai@uconn.edu (A.B.B.) \\ * Correspondence: carolyn.lin@uconn.edu
}

\begin{abstract}
Objective: The current study investigates the effects of an alcohol-prevention program delivered to college students in a formal classroom setting. Participants: The sample comprised 231 first-year college students who enrolled in a multisection "First Year Experience" course at a large northeastern university in the United States. Method: A naturalistic experiment was conducted, with a baseline evaluation at the beginning of the semester and a post-experiment evaluation near the end of the semester. Results: Social drinking attitudes, proximal drinking norm and the college effect are significant predictors of pre- and post-intervention episodic drinking frequency. The intervention reduced episodic drinking frequency as well as perceived distal and proximal drinking norms. It also increased drinking attitudes and did not change perceived efficacy or drinking-outcome expectancies. Conclusions: Practitioners could consider implementing a similar intervention to allow students to learn and practice safe drinking skills in the first year of their college life.
\end{abstract}

Keywords: adaptation strategies; alcohol prevention; college effect; naturalistic experiment; theory of planned behavior

\section{Introduction}

Episodic drinking behavior is a significant health problem on American college campuses. According to the National Survey on Drug Use and Health (NSDUH), 53 and 33\% of full-time U.S. college students between the ages of 18-22 reported regularly drinking alcoholic beverages and engaging in binge drinking, respectively (SAMHSA 2019). The U.S. health agency, National Institute on Alcohol Abuse and Alcoholism, defines binge drinking as a drinking pattern that raises the blood alcohol concentration of an adult to 0.08 percent (or $0.08 \mathrm{~g}$ of alcohol per deciliter) or higher, through consuming five or more drinks (male) and four or more drinks (female) in $2 \mathrm{~h}$ or less (NIAAA National Advisory Council 2004). Unintended consequences of binge drinking among American college students include motor-vehicle crashes, campus violence, sexual assaults and date rape, psychological problems, as well as academic difficulties such as missing and failing classes and overall lower grades (Blanco et al. 2008; Hingson et al. 2009; Wechsler et al. 2000; Ehlers et al. 2018; Miller et al. 2017; Gilmore et al. 2015; Ehlke et al. 2021; An et al. 2017).

The present study explored the influence of the college drinking culture, alongside other behavioral and social influence factors on students' alcohol consumption behavior. Specifically, this study implemented a naturalistic experiment with a college freshman population via a baseline evaluation at the beginning of the autumn semester, an alcoholprevention program as intervention and a post-experiment evaluation at the end of the semester. To examine the role that alcohol-use cognition, attitude, expectancy and culture play on impacting student ability to control their drinking behavior, the study's conceptual framework is guided by the constructs of theory of planned behavior, outcome expectancies, social norms and college effect (or the drinking culture in a developmental phase). 


\subsection{College Effect}

Many college students experience developmentally limited alcoholism, which is characterized by the transition from embracing their peers-and abandoning their parents-as the major agents of influence (Ichiyama and Kruse 1998; Zucker 1994). Research suggests that alcohol dependence during this age-related transitional period could be considered a "short-duration" or "chronic and episodic" phenomenon (Vergés et al. 2012). This type of life-stage specific alcoholism has been found to coincide with the "college effect," which is marked by favorable beliefs, motivations and attitudes toward experiencing their "rite of passage" with drinking and partying among college freshmen (Lin and Carlson 2009).

The concept of "college effect" (Lin and Carlson 2009) can hence be conceptualized as a form of social learning, where new students model their more senior counterparts' behaviors such as excessive drinking and partying to maximize enjoyment of their college experience. Specifically, the process of new students identifying themselves with peers that favor heavy episodic drinking behavior is consistent with Bandura's (1969) concept of reciprocal determinism, a dimension of social learning theory, which suggests that a reciprocal process of influence will take place between individuals and their environment.

Arnett (2000) introduced the term "emerging adulthood" to conceptually define the period of transition between adolescence and adult roles. Emerging adults, especially those who have just left high school and entered college life, are highly susceptible to the college effect described above, as they often view drinking as an important part of their college experience (NIAAA 2021). The college effect, as conceptualized here, could hence pose a direct challenge to the student health objective of reducing heavy alcohol consumption and binge drinking on college campuses. As little empirical research has examined the college effect concept, the following research hypotheses are proposed to validate this phenomenon in the current study context.

Hypothesis 1a (H1a). The college effect will be a positive predictor of drinking frequency at pre-intervention phase.

Hypothesis $\mathbf{1 b}(\mathbf{H} 1 \mathbf{b})$. The college effect will be a positive predictor of drinking frequency at post-intervention phase.

Within this "college effect" framework, the discussion below presents the relevant cognitive and affective constructs developed based on the theory of planned behavior (Ajzen 1991, 2005). The theory of planned behavior (TPB) postulates that the intention to perform a behavior is determined by attitudes towards the behavior, social norms concerning the performance of the behavior and perceived behavioral control (Ajzen 1991, 2005).

\subsection{Social Drinking Attitudes}

Ajzen (1991) conceptualizes attitudes as the degree to which one evaluates a behavior as positive or negative. Specifically, if an individual believes that the outcome of performing a specific behavior is positive, then the individual would hold a positive attitude toward the behavior and vice versa (Ajzen 1991, 2005; Montano and Kasprzyk 2015). Ajzen (1991) also considers that an individual's beliefs about a behavior are formed by associating the behavior with certain characteristics, attributes, events or other behaviors.

Past studies have found that positive attitudes toward alcohol consumption were significant predictors of alcohol use among college students (Bravo et al. 2017; Huchting et al. 2008; Cooke et al. 2016; Trafimow 1996; DiBello et al. 2018; Dormal et al. 2018). Other researchers have also reported that college students' attitudes toward binge drinking were a significant positive predictor of binge drinking intentions (Elliott and Ainsworth 2012; Johnston and White 2003; Norman et al. 2007; Norman 2011; DiBello et al. 2018; Dormal et al. 2018; Willis et al. 2020). 
Additional studies have likewise suggested that students' positive attitudes toward engaging in binge drinking were the strongest predictors of binge drinking intentions when compared to the other variables of the TPB paradigm (e.g., subjective norms and perceived behavioral control) (Norman et al. 2007; Norman 2011; DiBello et al. 2018; Willis et al. 2020). Similarly, favorable attitudes toward alcohol use were significant and positive predictors of drinking among college students affiliated with a college fraternity organization (Huchting et al. 2008). To validate the theoretical link between attitude toward drinking and drinking behavior in the current study context, the following hypotheses are tested.

Hypothesis 2a (H2a). Attitudes toward alcohol use will be a positive predictor of drinking frequency at pre-intervention phase.

Hypothesis $\mathbf{2 b}(\mathbf{H} \mathbf{2 b})$. Attitudes toward alcohol use will be a positive predictor of drinking frequency at post-intervention phase.

\subsection{Social Drinking Norms}

Prior studies that focused on alcohol consumption among college students indicated a positive correlation between social norms regarding alcohol consumptions and intentions to use alcohol (Carcioppolo and Jensen 2012; Halim et al. 2012; DiBello et al. 2018; DiGuiseppi et al. 2018a, 2018b; DiGuiseppi et al. 2020). Previous studies have also demonstrated that college students tend to over-represent alcohol consumption among their peers (Borsari and Carey 2001; Carcioppolo and Jensen 2012; Halim et al. 2012; LaBrie et al. 2010; Kenney et al. 2017; Cox et al. 2019). The construct of social norms in the TPB framework is operationalized as subjective norms to reflect an individual's beliefs about whether referent important others would approve or disapprove the individual's decision of engaging in a specific behavior (Ajzen 1991).

In a meta-analysis evaluating the efficacy of the TPB, Armitage and Conner (2001) reported that subjective norms were the weakest predictor of behavioral intentions. Another meta-analytic review similarly suggested that subjective norms explained little to no variance in behavioral intentions (Hagger et al. 2002). DiBello et al. (2018) and Willis et al. (2020) tested the predictive strength of injunctive norms (considered analogous to subjective norms) and descriptive norms; their findings revealed that only descriptive norms significantly predicted alcohol use among college students. Cialdini et al. (1990) conceptualized descriptive social norms as an individual's perception regarding the behaviors of others; they asserted that descriptive norms reflect the type of social norms developed through observation of public behaviors that are largely endorsed by a referent group.

Rivis and Sheeran's (2003) meta-analysis study validated the hypothesis that the relationship between descriptive norm and intention was positive and stronger among younger samples than samples of other age groups. This hypothesis was derived from life-span developmental psychology, which suggests that adolescents and young adults are more pressured to conform to real or perceived social norms to gain group acceptance (Mullen and Suls 1982; Pasupathi 1999; Castro et al. 1987; Van der Pligt and Eiser 1984). Previous research has also indicated that perceived alcohol use of proximal others (friends) and distal others (a typical student) had a significant impact on college students' drinking habits, such that students who perceived high drinking prevalence among their friends and student body in general were more likely to drink alcohol (Halim et al. 2012; Paschall et al. 2014; Kenney et al. 2017).

For example, past research has reported a high correlation $(r=0.70)$ between descriptive drinking norms and students' drinking behavior, when "friends" were considered a normative reference group, (Carey et al. 2006). Comparatively, the correlation $(r=0.40)$ was relatively lower between descriptive drinking norms and drinking behavior, when the "typical university student" was used as a normative reference group (Cho 2006). These findings were consistent with a meta-analysis study, which showed that students' perceptions of their own drinking frequency were closer to that of their close rather than 
distal others (Borsari and Carey 2003). Additional research also confirmed that students perceptions of proximal others' drinking behavior were more similar to their own drinking behavior, relative to their perceptions of the drinking behavior of "same-age" students (McAlaney and McMahon 2007).

Extant research that specifically focuses on assessing the independent contribution of proximal vs. distal descriptive norms on students' drinking behavior remains limited. To further validate whether perceptions of observed proximal vs. distal drinking norms influence students' drinking behavior, the following hypotheses are postulated.

Hypothesis 3a (H3a). Perceived descriptive proximal drinking norm will be a positive predictor of drinking frequency at pre-intervention phase.

Hypothesis $3 \mathbf{b}(\mathbf{H} 3 \mathbf{b})$. Perceived descriptive proximal drinking norm will be a positive predictor of drinking frequency at post-intervention phase.

Hypothesis 4a (H4a). Perceived descriptive distal drinking norm will be a positive predictor of drinking frequency at pre-intervention phase.

Hypothesis $\mathbf{4 b} \mathbf{b} \mathbf{H} \mathbf{4 b})$. Perceived descriptive distal drinking norm will be a positive predictor of drinking frequency at post-intervention phase.

\subsection{Controlled Drinking Efficacy}

An individual's success in performing a behavior could depend on how much the behavior is under his or her volitional control (Montano and Kasprzyk 2015). The TPB conceptualizes perceived behavioral control as an individual's perceived ease or difficulty of performing a behavior (Ajzen 2005). The concept of perceived behavioral control is different from Bandura's (1995) construct of perceived self-efficacy, as the former emphasizes the perceptions of control over a behavior and the latter focuses on the belief in one's confidence to perform a behavior (Cooke et al. 2016; Norman et al. 2007; Armitage and Conner 2001; Norman and Hoyle 2004).

In the context of alcohol research among college students, perceived behavioral control was conceptualized as students' perceptions of control regarding drinking and included both internal control factors (e.g., skills) and external control factors (e.g., environmental pressures) (Huchting et al. 2008; Cooke et al. 2016; Norman et al. 2007; Gabbiadini et al. 2017). Meta-analysis studies suggested that perceived behavioral control uniquely contributed to the prediction of both intentions and behavior (Cooke et al. 2016; Armitage and Conner 2001). For example, Willis et al. (2020) and Gabbiadini et al. (2017) found a significant negative relationship between perceived behavioral control and drinking intentions, such that students who reported lower perceived behavioral control were more likely to express intentions to consume alcohol.

Due to variations in operationalization, the direction of the relationship between perceived behavioral control and alcohol consumption also varies. A meta-analysis conducted by Cooke et al. (2016) demonstrated that these contradictory findings may be a result of student reaction to the "perceived behavioral control" measurement. Specifically, students may believe that alcohol consumption is a volitional action, as they underestimate the impact of external factors (i.e., peer pressure) on their drinking behavior. To investigate whether students' beliefs about their ability to practice safe drinking skills influence their drinking behavior, the current study incorporated both internal and external factors to measure the proposed "controlled-drinking efficacy" construct. The following hypothesis are posited to test the role of controlled-drinking efficacy in students' drinking behavior.

Hypothesis 5a (H5a). Perceived controlled-drinking efficacy will be a positive predictor of drinking frequency at pre-intervention phase. 
Hypothesis $\mathbf{5 b} \mathbf{b} \mathbf{H} \mathbf{b})$. Perceived controlled-drinking efficacy will be a positive predictor of drinking frequency at post-intervention phase.

\subsection{Social Drinking Outcome Expectancies}

Researchers suggested that a key factor in theories of health-related behaviors is people's cognitive motivation to engage in risky behaviors (Engels et al. 2005; Goldman et al. 1999). According to the alcohol expectancy theory, individuals' experiences (vicarious and direct) with alcohol consumption can influence their drinking-related outcome expectancies (Goldman et al. 1999). Researchers have explored the effects of positive alcohol expectancies such as mood enhancement, stress reduction, feelings of happiness, increased affective expression and social facilitation-as well as negative alcohol expectancies such as aggression, sadness and depression, physical illness, poor school performance and risk of motor vehicle crashes-on students' alcohol consumption (Rimal and Real 2005; Goldman et al. 1999; Leigh and Stacy 1993; Cashin et al. 1998; Ham 2009; Ham et al. 2016; Papachristou et al. 2018; Booth and Hasking 2009; Baines et al. 2016).

College students who reported higher levels of positive alcohol expectancies were more motivated to indicate higher levels of alcohol consumption (Leigh and Stacy 1993; Chassin and DeLucia 1996; Dunne and Katz 2015; LaBrie et al. 2008; Papachristou et al. 2018; Booth and Hasking 2009; Ham 2009; Ham et al. 2016; Baines et al. 2016). Similarly, studies have found a negative relationship between negative alcohol expectancies and drinking behaviors (Goldman et al. 1999; Goldberg et al. 2002; Goldman 1994; Papachristou et al. 2018; Ham et al. 2016). Longitudinal studies also confirmed positive and negative alcohol expectancies as predictors of drinking behaviors and drinking levels among young adults (Goldman et al. 1999; Christiansen et al. 1989).

In a recent meta-analysis of alcohol interventions targeting college fraternity-society students in the U.S., Scott-Sheldon et al. (2016) found that challenging students' alcohol expectancies could lead to reduction in alcohol consumption on episodic drinking occasions, such as parties or weekend drinking. As more research is still needed to explore how perceived positive drinking outcomes associated with social-interaction expectancy could influence students' drinking behavior, the current study chose to examine the connection between these two variables before and after intervention via the following hypotheses.

Hypothesis 6a (H6a). Perceived positive social-drinking outcomes will be a positive predictor of drinking frequency at pre-intervention phase.

Hypothesis $\mathbf{6 b} \mathbf{b} \mathbf{H 6 b})$. Perceived positive social-drinking outcomes will be a positive predictor of drinking frequency at post-intervention phase.

Lastly, to compare the pre-intervention and post-intervention evaluation results across all measures, a research question is posed below:

RQ1a-f: Will pre-intervention and post-intervention scores differ with respect to (a) drinking attitudes, (b) perceived proximal drinking norms, (c) perceived distal drinking norms (d) controlled drinking efficacy, (e) drinking outcome expectancies and (f) drinking frequency?

\section{Materials and Methods}

This study was conducted at a large northeastern university in the U.S, where highrisk drinking behavior has been an ongoing phenomenon for a number of years. The study sample was represented by newly arrived students who enrolled in a multisection "First Year Experience" course, with the cooperation and approval of the offices of the vice provost for undergraduate studies and vice president of student affairs. The objectives of the course are to help facilitate student transition to college life and to build a foundation for developing a successful college career. Prior to sample recruitment, the study protocol was approved by the Institutional Review Board that evaluates protection for human subjects in research. A naturalistic experiment was conducted with these students in a 
classroom setting, in addition to a baseline evaluation at the beginning of the semester and a post-experiment evaluation near the end of the semester. These evaluation surveys were conducted online via a hyperlink embedded in the course website.

This naturalistic experiment incorporated the research stimulus as part of the course topic that addressed alcohol in college life. The stimulus consisted of introducing what constitutes a standard drink for different types of alcoholic beverages and class presentations of a three-part binge-drinking prevention program. These programs include (1) Make A Drink: having the students pour the "pretend" 80-proof alcohol that they would typically consume into a solo cup and then have them pour the liquid into shot glasses to demonstrate how many shots are actually in that solo cup; (2) What Happens: showing the physiological effects (e.g., brain, heart and other health conditions), physical outcomes (e.g., physical fights, injuries and unprotected sex) and legal ramifications (e.g., university sanctions and local/state laws) of unsafe drinking behavior; (3) Down More Drinks: having students engage in a role-playing game to practice controlled-drinking skills at a social event, when pressured by other students to participate in unsafe drinking behavior.

The total number of valid survey responses was 612 for the baseline evaluation and 465 for post-experiment evaluation. Using a systematic data cleaning procedure, only those matched cases without any data entry errors were retained. These error-free cases $(\mathrm{N}=231)$, containing the matched responses between the baseline and post-experiment evaluations, were utilized for data analysis. The $24 \%$ attrition rate for the study (which tested more than 30 measurement items) is below the critical threshold of $40 \%$ random attrition rate deemed necessary to maintain data validity for a college student sample in a repeated measure study (Pan and Zhan 2020).

\subsection{Definitions}

The survey instrument contains the following measurement items that operationally defined a set of theoretical concepts explicated above and were used to test the proposed research hypotheses and questions. All scale items were measured on a 7-point scale (e.g., " 7 " = strongly agree and " 1 " = strongly disagree), unless otherwise specified.

\subsubsection{Drinking Attitudes}

Five items were used to assess the degree to which one evaluates drinking behavior as positive or negative. Sample items included: (1) getting sick from drinking is the necessary price to pay for having fun; (2) you won't get alcohol poisoning, even if you do get drunk. Responses to the five items were averaged to form the drinking attitudes variable, with larger values indicating more positive attitudes towards episodic social drinking (pretest, $\alpha=0.75$; post-test, $\alpha=0.82$ ).

\subsubsection{Drinking Norms}

Two separate items were utilized to assess perceived distal and proximal socialdrinking norms. For distal norm, participants were asked to estimate the percentage of students at their university who: (1) drink at least once a month and (2) drink at least once a week. For proximal norm, they were also prompted to offer the percentage of their friends who: (1) drink at least once a month and (2) drink at least once a week. For each item, participants reported a percentage between 0 and 100 (including $0,1-9,10-19 \%$ and so on). Responses to these two sets of items were each averaged to create separate measures of distal and proximal drinking norms.

\subsubsection{Controlled Drinking Efficacy}

Twelve items were developed to measure students' perceived ability to control their drinking behavior at a social drinking occasion. These items include: (1) control oneself to reduce alcohol consumption: (2) determine the proper amount of alcohol to consume safely; (3) only have medically safe drinks; (4) watch self to not over drink; (5) have nonalcoholic beverages to balance the alcohol intake: (6) monitor physical reactions to adjust alcohol 
intake; (7) resolve not to drink to get drunk; (8) stretch the amount of time downing each drink; (9) control the number of drinks for a specific time duration; (10) monitor one's mood to control alcohol intake; (11) say no to your friends if they pressure you to keep drinking; (12) stop drinking alcohol even when your friends are still drinking. Responses to the twelve items were averaged to form the controlled-drinking efficacy variable, with larger values indicating a greater efficacy to practice safe-drinking skills (pretest, $\alpha=0.98$; post-test, $\alpha=0.99$ ).

\subsubsection{Drinking Outcome Expectancies}

Individuals' beliefs about the anticipated consequences of drinking alcohol were measured by seven items. Participants were asked to indicate the degree to which they believed various social-interaction outcomes would occur in a social drinking context. Sample items included "drinking alcohol can make you feel more physically attractive" and "drinking alcohol can make you overcome your shyness." Responses to the seven items were averaged to form a measure of social-drinking outcome expectancies, with larger values indicating more desirable expectations regarding episodic social drinking outcomes (pretest, $\alpha=0.83$; post-test, $\alpha=0.90$ ).

\subsubsection{College Effect}

At baseline, participants were asked to indicate whether a set of activities reflects their college culture evolving around alcohol use. Six measurement items were developed based on the conceptualization proposed by Lin and Carlson (2009). These items included: drinking alcohol anytime they want; drinking as much alcohol as they want; going to parties to drink alcohol, playing drinking games, partying as much as they can; getting drunk with their friends as a rite of passage. Summed scores for all items were averaged to construct a composite variable $(\alpha=0.95)$.

\subsubsection{Drinking Frequency}

Participants were asked to report the number of occasions they had a drink of alcohol in the past month. The response categories ranged from 0 occasions to 40 or more occasions (measured via 7 incremental levels), with a higher value indicating a greater drinking frequency.

\section{Results}

Table 1 presents the Pearson product-moment correlations across all study variables. It also displays the means and standard deviations for all the paired pre- and post-intervention variables.

Table 1. Zero-order correlations, means and standard deviations.

\begin{tabular}{|c|c|c|c|c|c|c|c|c|c|c|c|c|c|}
\hline & 1 & 2 & 3 & 4 & 5 & 6 & 7 & 8 & 9 & 10 & 11 & 12 & 13 \\
\hline 1 College effect & - & & & & & & & & & & & & \\
\hline 2 Attitudes (Pre) & $0.66 * *$ & & & & & & & & & & & & \\
\hline 3 Attitudes (Post) & $0.47^{* *}$ & $0.63 * *$ & & & & & & & & & & & \\
\hline 4 Distal norms (Pre) & $0.19 * *$ & $0.18^{* *}$ & $0.14 *$ & & & & & & & & & & \\
\hline 5 Distal norms (Post) & 0.08 & 0.12 & 0.08 & $0.54^{* *}$ & & & & & & & & & \\
\hline 6 Proximal norms (Pre) & $0.49 * *$ & $0.41 * *$ & $0.29 * *$ & $0.35^{* *}$ & $0.19 * *$ & & & & & & & & \\
\hline 7 Proximal norms (Post) & $0.38^{* *}$ & $0.37 * *$ & $0.30 * *$ & $0.21 * *$ & $0.40 * *$ & $0.72 * *$ & & & & & & & \\
\hline 8 Control Efficacy (Pre) & -0.07 & -0.13 & -0.08 & -0.05 & 0.01 & -0.07 & -0.07 & & & & & & \\
\hline 9 Control Efficacy (Post) & -0.02 & -0.06 & -0.12 & 0.1 & $0.14 *$ & 0.03 & 0.01 & $0.43^{* *}$ & & & & & \\
\hline 10 Expectations (Pre) & $0.57^{* *}$ & $0.32 * *$ & $0.21^{* *}$ & $0.14^{*}$ & 0.06 & $0.42 * *$ & $0.35^{* *}$ & -0.12 & -0.04 & & & & \\
\hline 11 Expectations (Post) & $0.42 * *$ & $0.25^{* *}$ & 0.30 ** & 0.05 & 0.13 & $0.29 * *$ & $0.36^{* *}$ & -0.09 & -0.1 & $0.45 * *$ & & & \\
\hline 12 Drinking Freq (Pre) & $0.62 * *$ & $0.50 * *$ & $0.42 * *$ & $0.13^{*}$ & 0.09 & $0.57^{* *}$ & $0.50 * *$ & -0.01 & 0.02 & $0.58 * *$ & $0.45^{* *}$ & & \\
\hline 13 Drinking Freq (Post) & $0.51 * *$ & $0.41^{* *}$ & $0.47^{* *}$ & 0.1 & 0.13 & $0.50 * *$ & $0.52 * *$ & -0.03 & -0.03 & 0.55 & $0.61^{* *}$ & $0.76^{* *}$ & - \\
\hline$M$ & 2.29 & 2.2 & 2.57 & 69.57 & 63.44 & 60.21 & 55.52 & 5.48 & 5.35 & 1.24 & 1.27 & 3.19 & 2.76 \\
\hline$S D$ & 1.33 & 1.15 & 1.33 & 15.75 & 18.14 & 29.67 & 29.48 & 1.66 & 1.7 & 0.53 & 0.68 & 3.73 & 3.25 \\
\hline
\end{tabular}




\subsection{Pre- and Post-Intervention Models}

\subsubsection{Pre-Intervention Model}

Multiple linear regression was used to test the pre-intervention and post-intervention models (see Table 2). Attitudes, norms, efficacy and expectancies were entered as predictors of drinking frequency in the first step. The college effect variable was entered in the second step to examine whether it accounted for variance above and beyond the first set of predictors. Standardized regression coefficients are reported with alpha set to 0.05 . The pre-intervention model was significant at the first step, $F(5,204)=32.43, p<0.001\left(R^{2}=0.44\right.$; adjusted $\left.R^{2}=0.43\right)$. As hypothesized, attitudes $\left(B=0.31, p<0.001\right.$, Cohen's $\left.f^{2}=0.07\right)$ and proximal drinking norm $\left(B=0.46, p<0.001\right.$, Cohen's $\left.f^{2}=0.19\right)$ were positive predictors of drinking frequency, these findings thus confirmed $\mathrm{H} 2 \mathrm{a}$ and $\mathrm{H} 3 \mathrm{a}$, respectively. However, perceived distal drinking norm $\left(B=-0.08, p>0.10\right.$, Cohen's $\left.f^{2}=0.01\right)$, controlled drinking efficacy ( $B=0.08, p>0.10$, Cohen's $\left.f^{2}=0.01\right)$ and outcome expectancies $(B=0.08, p>0.10$, Cohen's $f^{2}=0.00$ ) were irrelevant to drinking frequency. These results rendered H4a, H5a and H6a without support.

Table 2. Final pre- and post-intervention regression models predicting drinking frequency.

\begin{tabular}{|c|c|c|c|c|c|c|c|c|}
\hline & $B$ & $S E$ & B & $t$ & VIF & $R^{2}$ & Adjusted $R^{2}$ & $F$ \\
\hline \multicolumn{2}{|c|}{ Pre-Intervention Model } & & & & & 0.51 & 0.50 & $35.24^{* * *}$ \\
\hline Attitudes & 0.50 & 0.22 & 0.15 & $2.27 * *$ & 1.88 & & & \\
\hline Norms (Distal) & -0.02 & 0.01 & -0.07 & -1.35 & 1.15 & & & \\
\hline Norms (Proximal) & 0.05 & 0.01 & 0.37 & $6.26^{* * *}$ & 1.47 & & & \\
\hline Efficacy & 0.15 & 0.12 & 0.07 & 1.33 & 1.04 & & & \\
\hline Expectancies & -0.05 & 0.17 & -0.02 & -0.29 & 1.63 & & & \\
\hline College Effect & 1.05 & 0.20 & 0.37 & $5.28^{* * *}$ & 2.07 & & & \\
\hline \multicolumn{2}{|c|}{ Post-Intervention Model } & & & & & 0.43 & 0.41 & $23.80^{* * *}$ \\
\hline Attitudes & 0.51 & 0.17 & 0.22 & $2.99 * *$ & 1.74 & & & \\
\hline Norms (Distal) & -0.01 & 0.01 & -0.04 & -0.69 & 1.26 & & & \\
\hline Norms (Proximal) & 0.04 & 0.01 & 0.35 & $5.33^{* * *}$ & 1.45 & & & \\
\hline Efficacy & 0.01 & 0.10 & 0.01 & 0.13 & 1.05 & & & \\
\hline Expectancies & 0.16 & 0.15 & 0.07 & 1.06 & 1.63 & & & \\
\hline College Effect & 0.61 & 0.16 & 0.25 & $3.83^{* * *}$ & 1.41 & & & \\
\hline
\end{tabular}

${ }^{*} p<0.05,{ }^{* *} p<0.01,{ }^{* * *} p<0.001$.

At step 2, the addition of the college effect variable yielded a significant mode as well, $F(6,204)=35.24, p<0.001\left(R^{2}=0.51\right.$; adjusted $\left.R^{2}=0.50\right)$, accounting for an additional $6.7 \%$ of the variance $(p<0.001)$ and with an identical set of significant and nonsignificant predictors relative to step 1 . Specifically, while attitudes $\left(B=0.15, p<0.05\right.$, Cohen's $\left.f^{2}=0.01\right)$ and proximal drinking norm $\left(B=0.37, p<0.001\right.$, Cohen's $\left.f^{2}=0.10\right)$ were positive predictors of drinking frequency, the same is not true for perceived distal drinking norm $(B=-0.07$, $p<0.10$, Cohen's $\left.f^{2}=0.00\right)$, controlled drinking efficacy $\left(\beta=0.07, p>0.10\right.$, Cohen's $\left.f^{2}=0.00\right)$ and outcome expectancies $\left(B=-0.02, p>0.10\right.$, Cohen's $\left.f^{2}=0.00\right)$. With the college effect variable emerging as a significant predictor of social drinking frequency $(B=0.37, p<0.001$, Cohen's f2 $=0.07$ ) at step 2, this particular result validated H1a.

\subsubsection{Post-Intervention Model}

Regression analysis revealed that the post-intervention model was also significant at the first step, $F(5,189)=23.89, p<0.001\left(R^{2}=0.39\right.$; adjusted $\left.R^{2}=0.37\right)$. The pattern of findings in this initial post-intervention analysis was identical to the findings obtained for the pre-intervention analysis. As hypothesized, attitudes $(B=0.29, p<0.001$, Cohen's $\left.f^{2}=0.06\right)$ and proximal drinking norm $\left(B=0.41, p<0.001\right.$, Cohen's $\left.f^{2}=0.14\right)$ were positive predictors of drinking frequency, providing statistical support for $\mathrm{H} 2 \mathrm{~b}$ and $\mathrm{H} 3 \mathrm{~b}$. Perceived distal drinking norm $\left(B=-0.05, p>0.10\right.$, Cohen's $\left.f^{2}=0.00\right)$, controlled drinking efficacy $\left(B=0.01, p>0.10\right.$, Cohen's $\left.f^{2}=0.00\right)$ and outcome expectancies $(B=0.12, p>0.10$, Cohen's 
$f^{2}=0.01$ ) were not associated with drinking frequency. Thus, results failed to confirm $\mathrm{H} 4 \mathrm{~b}$, $\mathrm{H} 5 \mathrm{~b}$ and $\mathrm{H} 6 \mathrm{~b}$.

After introducing the college effects variable at the second step, the final post-intervention model was also statistically significant, $F(6,188)=23.80, p<0.001\left(R^{2}=0.43\right.$; adjusted $\left.R^{2}=0.41\right)$, accounting for an additional $4.4 \%$ of the variance $(p<0.001)$. Attitudes $(B=0.22$, $p<0.01$, Cohen's $\left.f^{2}=0.03\right)$ and proximal drinking norm $(\beta=0.35, p<0.001$, Cohen's $f^{2}=0.09$ ) were again the positive predictors of drinking frequency. The opposite is true for perceived distal drinking norm $\left(B=-0.04, p<0.10\right.$, Cohen's $\left.f^{2}=0.00\right)$, controlled drinking efficacy ( $B=0.01, p>0.10$, Cohen's $\left.f^{2}=0.00\right)$ and outcome expectancies $(B=0.07, p>0.10$, Cohen's $f^{2}=0.00$ ). As the college effect variable was found to be a significant predictor of drinking frequency $\left(B=0.25, p<0.001\right.$, Cohen's $\left.f^{2}=0.05\right)$ at step 2 , this result provided support for H1b.

\subsection{Pre- and Post-Intervention Change}

We conducted a series of paired samples t-tests to examine RQ1a-1f, which address whether pre-intervention and post-intervention scores differed with respect to attitudes, proximal norms, distal norms, efficacy, expectancies and drinking frequency. Given that there were six tests in total, the alpha threshold was reduced to 0.01 (guided by the Bonferroni correction) to avoid overly liberal rejection of multiple null hypotheses. Results indicated a significant pre-post difference for four of the six measures. Beginning with our criterion variable, post-intervention social drinking frequency was significantly lower than pre-intervention social drinking frequency, $t(215)=2.49, p=0.01$, Cohen's $d=0.17$, indicating the overall success of the intervention. With regards to the proposed determinants of social drinking behavior, post-intervention attitudes were significantly higher than pre-intervention attitudes, $t(207)=4.87, p<0.001$, Cohen's $d=-0.34$. The postintervention proximal-drinking norm was significantly lower than the pre-intervention norm, $t(221)=3.16, p<0.01$, Cohen's $d=0.21$. Likewise, the post-intervention perceived distal drinking norm was significantly lower than the pre-intervention norm, $t(225)=5.62$, $p<0.001$, Cohen's $d=0.37$. Although post-intervention drinking-outcome expectancies were higher than pre-intervention drinking-outcome expectancies, the test did not reach statistical significance when applying the reduced alpha threshold, $t(209)=-2.47, p<0.05$, Cohen's $d=-0.17$. No change was observed when considering controlled-drinking efficacy, as post-intervention scores did not differ from pre-intervention scores, $t(204)=1.05, p>10$, Cohen's $d=0.07$. See Table 1 for means and standard deviations.

\subsection{Additional Post Hoc Exploratory Analyses}

Carcioppolo and Jensen (2012) differentiated between students' perceptions of a typical student's drinking behavior (i.e., descriptive norms) and students' perceptions regarding drinking traditions on college campuses (i.e., historical drinking norms). Their study found that both types of descriptive norms predicted drinking behavior, and historical drinking norms also moderated the relationship between descriptive norms and drinking behaviors. In the current study context, the college effect could be seen as an extension of the "historical drinking norms," as it reflects the drinking culture that the new college students will learn and experience. To further explore and elucidate the role of the college effect on students' drinking behavior, a moderation analysis was conducted to ascertain whether the college effect moderated the relations between drinking frequency and its predictor variables.

First, we tested the baseline data, which showed that the interaction between the college effect and proximal norms was statistically significant $(B=0.47, p<0.05$, Cohen's $\left.f^{2}=0.04\right)$. The full model accounted for $58 \%$ of the variance in drinking behavior, with approximately $1 \%$ of the variance attributed to the addition of the interaction term $(p<0.05)$. By comparing participants who scored low versus high in the college effect (e.g., one standard deviation below and above the mean), results showed that when the college effect was low, the relationship between proximal norms and drinking behavior was positive 
but weak $(p<0.001)$. By comparison, the same positive relationship was much stronger when the college effect was high $(p<0.001)$. This indicates that proximal drinking norms were associated with higher drinking frequency, especially for participants who scored high on the college effect. None of the other variables, including distal norms, drinking attitudes, controlled-drinking efficacy and drinking outcome expectancies in the model, had a significant interaction with the college effect to predict drinking $(p>0.10)$.

Next, we conducted a similar analysis on the follow-up data. An identical pattern of results was observed. The interaction between the college effect and proximal norms was statistically significant $\left(ß=0.47, p<0.01\right.$, Cohen's $\left.f^{2}=0.03\right)$. The full model accounted for $55 \%$ of the variance in drinking behavior, with approximately $1 \%$ attributed to the interaction $(p<0.01)$. Once again, the positive relationship between proximal norms and drinking behavior was weak $(p<0.05)$ when the college effect was low but gained in strength when the college effect was high $(p<0.001)$. None of the other variables in the follow-up model significantly interacted with the college effect to predict drinking frequency.

\section{Discussion}

This study is among the first to implement an alcohol prevention program that directly engages and trains students in safe-drinking skills via a naturalistic experiment in a formal classroom setting. To assess the outcomes of this intervention, which was tailored to target the first-year college students, a pre-test and post-test evaluation was conducted. Evaluation results suggested that the intervention was successful in reducing social drinking behavior. Specifically, drinking frequency (RQ1f), proximal drinking norm(RQ1b) and distal drinking norm (RQ1c) all showed a decrease from pre-test to post-test. However, social drinking attitudes (RQ1a) increased in their magnitude from pre- to post-intervention. No significant change from pre-test to post-test was found for controlled drinking efficacy (RQ1d) and drinking outcome expectancies (RQ1e).

One possible explanation for this increase in drinking attitudes is that the intervention might have created an unanticipated effect. That is, the intervention in fact provided the students a positive vantage point for assessing their drinking behavior from a safe-drinking perspective. This unanticipated effect, however, seems to have been counterbalanced by the reduced drinking frequency resulting from the intervention. It is also likely that the lack of change in positive drinking-outcome expectancies could also have helped diffuse the potential effects associated with the increase in drinking attitude. In other words, even though participants viewed alcohol use more favorably at post-test, such positive attitudes were not complemented by increased drinking frequency or expectations of achieving desirable social-interaction consequences (e.g., overcoming shyness and feeling more socially confident).

The success of the current intervention on decreasing students' drinking frequency and perceived proximal drinking norm indicates a preliminary validation of the techniques used to engage and train the students in the experiment. Specifically, this intervention includes having the first-year college students play the "make a drink" game to become aware of the amount of 80-proof alcohol (i.e., the number of "shots") they typically consume, when a solo cup is used as the vessel for serving alcoholic beverages. Results of this exercise almost always reveal that students severely underestimate the actual amount of alcohol they usually consume at a social event. As a practice, this intervention module is both fun and useful to undergraduate students.

A second intervention component involves showing students "what happens" in an experiential mock exercise that demonstrates how heavy drinking can cause physical, psychological and/or financial injuries, such as assaults, fights, accidents, sickness, alcohol poisoning, arrests and legal charges, among others (Blanco et al. 2008; Hingson et al. 2009; Wechsler et al. 2000). Even though all first-year college students received online alcohol prevention training via the AlcoholEdu course, it is not surprising to find that students lack 
a good understanding of the real negative consequences of unsafe drinking behaviors until they have personally experienced them.

The third component of the intervention allows students to roleplay different types of safe-drinking skills, including sipping, eating food before drinking and between drinks, taking breaks between sips, adding a soft drink to the alcoholic beverage, safeguarding the drink, staying with friends and alcohol refusal techniques, among others. Again, it is not uncommon to find that students are utterly unaware of these "common-sense" safety tips that are not difficult to acquire and can lead to good safe-drinking habits.

Turing to the pre-test and post-test regression models that tested the predictors of drinking frequency, both models generated an identical pattern of results entailing the same set of significant and nonsignificant predictors. Specifically, more positive social drinking attitudes $(\mathrm{H} 2 \mathrm{a}-\mathrm{b})$ were a significant predictor of greater drinking frequency last month, alongside college effect (H1a-b) and proximal norm (H3a-b). However, the same is not true for distal norms (H4a-b), controlled-drinking efficacy (H5a-b) and outcome expectancies (H6a-b), as they were not found to be significant predictors of drinking frequency. With regard to the post hoc moderator analysis, the college effect emerged as a positive moderator for the influence of proximal norms on drinking frequency. The college effect did not significantly moderate the influence of other predictor variables on drinking frequency, however.

As proximal norms instead of distal drinking norms were a significant predictor of the two criterion variables, pre- and post-intervention episodic drinking behavior, these findings confirmed the results of some past research and contrasted that of others (Halim et al. 2012; Campo et al. 2003; Granfield 2005; Licciardone 2003; Lewis and Neighbors 2004; Perkins and Craig 2006; Thombs et al. 2007). In essence, the findings suggested that while students' drinking frequency was significantly influenced by their friends' drinking behaviors, a typical peer's drinking behaviors at school was not relevant in this context. By implication, these findings may also provide a partial explanation for why some social norms campaigns that focused on correcting the perceived distal descriptive norms might have failed (Paschall et al. 2006). These contradictory findings are not necessarily detrimental to the validity of these social norms campaigns, as variations in study settings could also generate different study results.

Findings further indicated that social drinking outcome expectancies were irrelevant to drinking frequency for both pre- and post-intervention evaluations. These results are inconsistent with past research findings (e.g., Papachristou et al. 2018; Ham et al. 2016; Baines et al. 2016). This contradiction could have been a function of the measurement items used in the current study for assessing outcomes expectancies. Specifically, these measures gauged student anticipation of gaining the desired social status and sociability outcomes through consuming alcohol in a party setting. For this reason, participants might have been in denial of their reliance on alcohol to achieve their social interaction efficacy. To put it another way, if participants could acknowledge how they rely on heavy episodic drinking to feel more socially adequate or integrated, then this admission may help them consider such harmful consequences as drunken brawls, injury and unprotected sex more carefully.

Interestingly, results also showed that controlled-drinking efficacy was not a significant predictor of pre- and post-intervention drinking frequency. These findings may reflect an optimistic bias whereby students may believe that they are in control of their alcohol consumption, while overlooking external influences such as social drinking norms and the college effect. For example, according to Cooke et al. (2016), students may consider college drinking behavior as an outcome of their own volition, which is not subject to other environmental influences (e.g., peer pressure). Another plausible explanation could be that students who are regular episodic drinkers may envision themselves as having sufficient control over their alcohol consumption behavior, as they perceive negative drinking outcomes to be normal and accepted as part of the partying scene (Lin et al. 2014). It is also possible that as a majority of the students were engaged in episodic social drinking, 
the intervention may not have been compelling enough to change student belief about their own controlled-drinking efficacy.

As for the role of the college effect played in students' drinking behavior, the findings clearly suggested that the college-effect related mindset, which might have reflected a hedonistic drinking culture, was at display here (Bravo et al. 2017; Cooke et al. 2016; Norman et al. 2007; Norman 2011). As the results of the moderation analyses singled out the college effect as a positive moderator only between proximal norms and drinking frequency, this suggests that when the college effect was stronger, so was the impact of friends' drinking norms on social drinking frequency. These findings also broadly confirmed Carcioppolo and Jensen's (2012) conception of historical drinking norms, which describes a type of descriptive norms that is reflected in the drinking traditions on college campuses. Based on the findings associated with the college effect, a construct that is conceptualized to be related to developmental-stage attitudes toward alcohol use, it is clear that the college effect could be an important factor for motivating episodic drinking activity on college campuses.

It is worth noting that even though the constructs of attitudes, outcome expectancies and efficacy associated with alcohol-use behavior (Bravo et al. 2017; Huchting et al. 2008; Cooke et al. 2016) are not necessarily new, an innovative "reality-based" approach was adopted here to conceptualize these measures from the students' point of view. In particular, this approach invites students to evaluate their drinking attitudes to include a proxy rite of passage measure; it also enables students to express their drinking-outcome expectancies via the desired social interaction efficacy in a social setting. As such, the attitude measure reflects an air of invincibility and inevitability, as it relates to why one chooses to drink and enjoys alcohol use without a care in the world. In a similar vein, the construct of drinking outcome expectancies represents those positive social consequences that one would hope to experience. This conceptual approach is particularly suited for an intervention study that intends to make an impression on the students who need to know how to stay safe if they choose to drink alcohol at a party. By the same token, the construct of drinking efficacy is conceptualized as a "controlled" drinking efficacy. Through focusing on the "controlled" aspect of drinking behavior, this construct again enables the intervention to emphasize the attainable safe-drinking skills that students could learn to practice at a social drinking event.

As the current study reconceptualized the construct of drinking attitudes, outcome expectancies and self-control efficacy through a "safe-drinking" intervention, it also introduced the college effect construct stemming from the framework of developmental-stage alcoholism. Under this framework, an individual does not typically evaluate the negative outcomes or implications of episodic alcohol use, social drinking norms or self-control efficacy to manage their drinking behavior for harm avoidance in a rational manner. Hence, the underlying theoretical assumptions linking all the variables under study give permission to an alternative approach that is distinct from the "rational decision-making" paradigm articulated by the TPB. This alternative approach instead assesses, explains and intervenes in the unsafe drinking behavior from the standpoint of a hedonistic drinking culture (Bravo et al. 2017; Cooke et al. 2016; Norman et al. 2007; Norman 2011), consistent with the historical drinking norms (Carcioppolo and Jensen 2012) and the characteristics of the college effect.

Taken together, these study findings suggest that when the first-year college students attend a social event, they choose to consume alcohol because drinking is an important part of college culture. These college students are also willing to accept both the good (e.g., being social) and the bad (e.g., feeling sick from over drinking) outcomes associated with the college effect, since attending college is a once in a lifetime opportunity. As these college students' alcohol consumption activities fall into a form of developmental alcoholism, they do not necessarily internalize the "locus of control" that will help guide them to develop meaningful forethoughts to rationally evaluate the risks and benefits of the real-world consequences. Hence, when addressing the episodic heavy-drinking 
problem among college students, it would be useful to consider the cognitive, affective and behavioral aspects of this phenomenon from the students' perspective. This approach would better enable researchers and practitioners alike to develop evidence-based tailored communication strategies to more effectively target these emerging adults with achievable and desirable positive health outcomes.

\subsection{Study Limitations}

Several limitations are noted for this study, as follows. First, the current research tested a conceptual framework with selective measures to validate the experimental results. Additional measures related to other developmental factors such as emotional and personality factors could be added as potential moderators to explore more advanced theoretical explanations. Second, a set of measures was developed to test both existing and new theoretical concepts that were incorporated in an innovative study framework. Even though these measures all exhibited satisfactory measurement validity and reliability, further refinement could potentially improve their replicability in relevant studies that are conducted with a similar or different methodology. Third, study results were generated through a pre- and post-measure one-shot field experimental design. To verify these findings, longitudinal studies that replicate the current research design would be required.

\subsection{Implications for Research and Best Practices}

While the intervention did not generate a statistically significant result in altering student attitudes or expectancies toward alcohol consumption, these results are not wholly unexpected for exposure to a one-shot only intervention. Realistically speaking, changing student attitudes toward alcohol use is not a goal that is attainable through this type of oneshot intervention, as alcohol consumption has been both widely prevalent and celebrated in American society and Western culture for centuries.

Importantly, the finding that indicates a decrease in student perception of other students' drinking behavior, including both perceived distal and proximal drinking norm, is a highly significant outcome of the intervention. As perceived descriptive norms associated with alcohol use are both ascribed by the strength of students' belief as well as reflected by the prevailing episodic drinking routine on campus, a change in perceived descriptive drinking norms may signal a potential conversion to endorse a lower social drinking frequency in the future.

The failure of the intervention to significantly increase student belief about their ability to practice safe-drinking skills was, again, not entirely unanticipated. Being acquainted with safe-drinking skills does not guarantee the application of these skills when students are participating in a party and wish to act the way that their friends and peers do. This is especially true, as the college effect could make students feel compelled to consume alcohol the way others do, for the purpose of demonstrating their willingness to "be social" at parties and gaining acceptance to establish their group identity. This type of thinking and reality then brings forth a challenge for the dominant alcohol-prevention paradigm, which emphasizes "teaching" and "correcting" students' misperceptions about the exaggerated student drinking norms.

Compared to recent research that investigated the effectiveness of mandatory alcohol prevention programs (implemented as a self-administered online alcohol education course), the current prevention approach is either equally or more effective in reducing the cognitive, affective and/or behavioral outcomes of episodic drinking behavior among the first-year students. For instance, an online prevention program such as Alcohol-Wise was found to reduce drinking frequency in a two-campus study, though additional research is needed to validate the study findings (Croom et al. 2015). However, the most widely administered online prevention program, AlcoholEdu, has continued to receive mixed results. For example, a study examining the impact of the course on reducing first-year drinking frequency found no support for its effectiveness (Paschall et al. 2014), as another study reported contradictory results (Lovecchio et al. 2010). 
Future research could consider developing alternative measurement items to assess the constructs that have been commonly used to discover potentially new and theoretically meaningful conceptual dimensions. For instance, a further exploration of the college effect construct may yield additional insights that are useful but currently unavailable. Empirical research should also consider applying the developmental-stage alcoholism perspective to study heavy episodic college drinkers to develop tailored and timely intervention, as these heavy drinkers may not mature out of their alcohol abuse after graduation.

In addition, adopting new communication technology such as a mobile phone app platform could be a productive approach to collect real-time and longitudinal data. For example, Merrill et al. (2018) utilized mobile texting in a pilot experiment to examine drinking behaviors, norms (injunctive and descriptive) and consequences. Study participants rated this messaging approach, which sent multiple text messages to their phone, to be highly acceptable.

Future studies could also consider developing a mobile phone app that would allow students to continuously interact with a set of features/icons to report their mood state and alcohol-use related activities. These types of data could be calibrated to prompt the mobile app to provide a real-time warning when the student is on the verge of entering a high-risk state emotionally and/or behaviorally. The data collected via the mobile app could also be applied as a self-reflection tool and incorporated into a one-on-one intervention session such as a motivational interview.

For practitioners, the one-shot intervention reported here serves as an example of an alternative intervention approach, relative to the conventional intervention strategy which often places its emphasis on broadly focused social norms campaigns, online training modules (e.g., AlcoholEdu) or judicially sanctioned education programs (e.g., BASICS). In particular, this multistage training routine, aiming at harm avoidance and harm reduction, could be easily implemented through interacting with students who share the same living quarters or student club membership in a group setting. Practitioners could consider implementing similar or the same set of intervention techniques (see presented here) to invite students to learn and practice safe-drinking skills in the first year of their college life.

Author Contributions: Conceptualization, C.A.L.; methodology, C.A.L.; validation, C.A.L., J.L.C. and A.B.B.; formal analysis, J.L.C. and C.A.L.; writing-original draft preparation, C.A.L., J.L.C. and A.B.B.; writing-review and editing, C.A.L., J.L.C. and A.B.B.; project administration, C.A.L. All authors have read and agreed to the published version of the manuscript.

Funding: This research received no external funding.

Institutional Review Board Statement: The study was conducted in accordance with the Declaration of Helsinki, and approved by the Institutional Review Board (or Ethics Committee) of University of Connecticut (protocol code H07-155, approved 25 June 2009).

Informed Consent Statement: Informed consent was obtained from all subjects involved in the study.

Data Availability Statement: The data are available upon request.

Conflicts of Interest: The authors declare no conflict of interest.

\section{References}

Ajzen, Icek. 1991. The theory of planned behavior. Organizational Behavior and Human Decision Processes 50: 179-211. [CrossRef] Ajzen, Icek. 2005. Attitudes, Personality, and Behavior. London: McGraw-Hill Education (UK).

An, Brian P., Chad N. Loes, and Teniell L. Trolian. 2017. The relation between binge drinking and academic performance: Considering the mediating effects of academic involvement. Journal of College Student Development 58: 492-508. [CrossRef]

Armitage, Christopher J., and Mark Conner. 2001. Efficacy of the theory of planned behaviour: A meta-analytic review. British Journal of Social Psychology 40: 471-99. [CrossRef]

Arnett, Jeffrey Jensen. 2000. Emerging adulthood: A theory of development from the late teens through the twenties. American Psychologist 55: 469. [CrossRef]

Baines, Laura, Andrew Jones, and Paul Christiansen. 2016. Hopelessness and alcohol use: The mediating role of drinking motives and outcome expectancies. Addictive Behaviors Reports 4: 65-69. [CrossRef] [PubMed] 
Bandura, Albert. 1969. Social learning of moral judgments. Journal of Personality and Social Psychology 11: 275-79. [CrossRef]

Bandura, Albert. 1995. Self-Efficacy in Changing Societies. Cambridge: Cambridge University Press.

Blanco, Carlos, Mayumi Okuda, Crystal Wright, D. S. Hasin, B. F. Grant, S. M. Liu, and M. Olfson. 2008. Mental health of college students and their non-college-attending peers: Results from the national epidemiologic study on alcohol and related conditions. Archives of General Psychiatry 65: 1429-37. [CrossRef]

Booth, Catherine, and Penelope Hasking. 2009. Social anxiety and alcohol consumption: The role of alcohol expectancies and reward sensitivity. Addictive Behaviors 34: 730-36. [CrossRef]

Borsari, Brian, and Kate B. Carey. 2001. Peer influences on college drinking: A review of the research. Journal of Substance Abuse 13: 391-424. [CrossRef]

Borsari, Brian, and Kate B. Carey. 2003. Descriptive and injunctive norms in college drinking: A meta-analytic integration. Journal of Studies on Alcohol 64: 331-41. [CrossRef]

Bravo, Adrian J., Mark A. Prince, and Matthew R. Pearson. 2017. College-related alcohol beliefs and problematic alcohol consumption: Alcohol protective behavioral strategies as a mediator. Substance Use and Misuse 52: 1059-68. [CrossRef] [PubMed]

Campo, Shelly, Dominique Brossard, M. Somjen Frazer, Timothy Marchell, Deborah Lewis, and Janis Talbot. 2003. Are social norms campaigns really magic bullets? Assessing the effects of students' misperceptions on drinking behavior. Health Communication 15: 481-97. [CrossRef]

Carcioppolo, Nick, and Jakob D. Jensen. 2012. Perceived historical drinking norms and current drinking behavior: Using the theory of normative social behavior as a framework for assessment. Health Communication 27: 766-75. [CrossRef] [PubMed]

Carey, Kate B., Brian Borsari, Michael P. Carey, and Stephen A. Maisto. 2006. Patterns and importance of self-other differences in college drinking norms. Psychology of Addictive Behaviors 20: 385-93. [CrossRef]

Cashin, Jeffrey R., Cheryl A. Presley, and Philip W. Meilman. 1998. Alcohol use in the Greek system: Follow the leader? Journal of Studies on Alcohol 59: 63-70. [CrossRef] [PubMed]

Castro, Felipe G., Ebrahim Maddahian, Michael D. Newcomb, and Peter M. Bentler. 1987. A multivariate model of the determinants of cigarette smoking among adolescents. Journal of Health and Social Behavior 28: 273-89. [CrossRef]

Chassin, Laurie, and Christian DeLucia. 1996. Drinking during adolescence. Alcohol Health and Research World 20: 175.

Cho, Hyunyi. 2006. Influences of norm proximity and norm types on binge and non-binge drinkers: Examining the under-examined aspects of social norms interventions on college campuses. Journal of Substance Use 11: 417-29. [CrossRef]

Christiansen, Bruce A., Gregory T. Smith, Patricia V. Roehling, and Mark S. Goldman. 1989. Using alcohol expectancies to predict adolescent drinking behavior after one year. Journal of Consulting and Clinical Psychology 57: 93. [CrossRef]

Cialdini, Robert B., Raymond R. Reno, and Carl A. Kallgren. 1990. A focus theory of normative conduct: Recycling the concept of norms to reduce littering in public places. Journal of Personality and Social Psychology 58: 1015. [CrossRef]

Cooke, Richard, Mark Dahdah, Paul Norman, and David P. French. 2016. How well does the theory of planned behaviour predict alcohol consumption? A systematic review and meta-analysis. Health Psychology Review 10: 148-67. [CrossRef]

Cox, Melissa J., Angelo M. DiBello, Matthew K. Meisel, Miles Q. Ott, Shannon R. Kenney, Melissa A. Clark, and Nancy P. Barnett. 2019. Do misperceptions of peer drinking influence personal drinking behavior? Results from a complete social network of first-year college students. Psychology of Addictive Behaviors 33: 297-303. [CrossRef]

Croom, Katherine, Lisa Staiano-Coico, Martin L. Lesser, Deborah K. Lewis, Valerie F. Reyna, Timothy C. Marchell, Jeremy Frank, and Stephanie Ives. 2015. The glass is half full: Evidence for efficacy of alcohol-wise at one university but not the other. Journal of Health Communication 20: 627-38. [CrossRef] [PubMed]

DiBello, Angelo M., Mary Beth Miller, Clayton Neighbors, Allecia Reid, and Kate B. Carey. 2018. The relative strength of attitudes versus perceived drinking norms as predictors of alcohol use. Addictive Behaviors 80: 39-46. [CrossRef] [PubMed]

DiGuiseppi, Graham T., Jordan P. Davis, Matthew K. Meisel, Melissa A. Clark, Mya L. Roberson, Miles Q. Ott, and Nancy P. Barnett. 2020. The influence of peer and parental norms on first-generation college students' binge drinking trajectories. Addictive Behaviors 103: 106227. [CrossRef]

DiGuiseppi, Graham T., Matthew K. Meisel, Sara G. Balestrieri, Miles Q. Ott, Melissa A. Clark, and Nancy P. Barnett. 2018a. Relationship between social network characteristics, alcohol use, and alcohol-related consequences in a large network of first-year college students: How do peer drinking norms fit in? Psychology of Addictive Behaviors 32: 914-21. [CrossRef]

DiGuiseppi, Graham T., Matthew K. Meisel, Sara G. Balestrieri, Miles Q. Ott, Melissa J. Cox, Melissa A. Clark, and Nancy P. Barnett. 2018b. Resistance to peer influence moderates the relationship between perceived (but not actual) peer norms and binge drinking in a college student social network. Addictive Behaviors 80: 47-52. [CrossRef]

Dormal, Valérie, Pierre Maurage, Séverine Lannoy, and Fabien D'Hondt. 2018. Positive attitude toward alcohol predicts actual consumption in young adults: An ecological implicit association test. Journal of Studies on Alcohol and Drugs 79: 733-40. [CrossRef] [PubMed]

Dunne, Eugene M., and Elizabeth C. Katz. 2015. Alcohol outcome expectancies and regrettable drinking-related social behaviors. Alcohol and Alcoholism 50: 393-98. [CrossRef] [PubMed]

Ehlers, Cindy L., Derek Wills, and David A. Gilder. 2018. A history of binge drinking during adolescence is associated with poorer sleep quality in young adult Mexican Americans and American Indians. Psychopharmacology 235: 1775-82. [CrossRef]

Ehlke, Sarah J., Michelle L. Kelley, and Abby L. Braitman. 2021. Weekly drinking and binge drinking mediate the association between drinking location and sexual coercion. Journal of Interpersonal Violence 36: 10843-62. [CrossRef] [PubMed] 
Elliott, Mark A., and Kirsty Ainsworth. 2012. Predicting university undergraduates' binge-drinking behavior: A comparative test of the one-and two-component theories of planned behavior. Addictive Behaviors 37: 92-101. [CrossRef] [PubMed]

Engels, Rutger C., Reinout Wiers, Lex E. Lemmers, and Geertjan Overbeek. 2005. Drinking motives, alcohol expectancies, self-efficacy, and drinking patterns. Journal of Drug Education 35: 147-66. [CrossRef] [PubMed]

Gabbiadini, Alessandro, Francesca Cristini, Luca Scacchi, and Maria G. Monaci. 2017. Testing the model of goal-directed behavior for predicting binge drinking among young people. Substance Use and Misuse 52: 493-506. [CrossRef]

Gilmore, Amanda K., Melissa A. Lewis, and William H. George. 2015. A randomized controlled trial targeting alcohol use and sexual assault risk among college women at high risk for victimization. Behavior Research and Therapy 74: 38-49. [CrossRef] [PubMed]

Goldberg, Julie H., Bonnie L. Halpern-Felsher, and Susan G. Millstein. 2002. Beyond invulnerability: The importance of benefits in adolescents' decision to drink alcohol. Health Psychology 21: 477. [CrossRef] [PubMed]

Goldman, Mark S. 1994. The alcohol expectancy concept: Applications to assessment, prevention, and treatment of alcohol abuse. Applied and Preventive Psychology 3: 131-44. [CrossRef]

Goldman, Mark S., Frances K. Del Boca, and Jack Darkes. 1999. Alcohol expectancy theory: The application of cognitive neuroscience. In The Guilford Substance Abuse Series. Psychological Theories of Drinking and Alcoholism. New York: The Guilford Press, pp. 203-46.

Granfield, Robert. 2005. Alcohol use in college: Limitations on the transformation of social norms. Addiction Research and Theory 13: 281-92. [CrossRef]

Hagger, Martin, Nikos Chatzisarantis, and Stuart Biddle. 2002. A meta-analytic review of the theories of reasoned action and planned behavior in physical activity: Predictive validity and the contribution of additional variables. Journal of Sport and Exercise Psychology 24: 3-32. [CrossRef]

Halim, Andrew, Penelope Hasking, and Felicity Allen. 2012. The role of social drinking motives in the relationship between social norms and alcohol consumption. Addictive Behaviors 37: 1335-41. [CrossRef]

Ham, Lindsay. S. 2009. Positive social alcohol outcome expectancies, social anxiety, and hazardous drinking in college students. Cognitive Therapy and Research 33: 615-23. [CrossRef]

Ham, Lindsay S., Amy K. Bacon, Maureen H. Carrigan, Byron L. Zamboanga, and Hilary G. Casner. 2016. Social anxiety and alcohol use: The role of alcohol expectancies about social outcomes. Addiction Research and Theory 24: 9-16. [CrossRef]

Hingson, Ralph W., Wenxing Zha, and Elissa R. Weitzman. 2009. Magnitude of and trends in alcohol-related mortality and morbidity among US college students ages 18-24, 1998-2005. Journal of Studies on Alcohol and Drugs 16: 12-20. [CrossRef]

Huchting, Karen, Andrew Lac, and Joseph W. LaBrie. 2008. An application of the theory of planned behavior to sorority alcohol consumption. Addictive Behaviors 33: 538-51. [CrossRef]

Ichiyama, Michael A., and Marc I. Kruse. 1998. The social contexts of binge drinking among private university freshmen. Journal of Alcohol and Drug Education 44: 1-8.

Johnston, Kim L., and Katherine M. White. 2003. Binge-drinking: A test of the role of group norms in the theory of planned behaviour. Psychology and Health 18: 63-77. [CrossRef]

Kenney, Shannon R., Miles Ott, Matthew K. Meisel, and Nancy P. Barnett. 2017. Alcohol perceptions and behavior in a residential peer social network. Addictive Behaviors 64: 143-47. [CrossRef]

LaBrie, Joseph, Toby Lamb, and Eric Pedersen. 2008. Changes in drinking patterns across the transition to college among first-year college males. Journal of Child and Adolescent Substance Abuse 18: 1-5. [CrossRef] [PubMed]

LaBrie, Joseph W., Justin F. Hummer, Sean Grant, and Andrew Lac. 2010. Immediate reductions in misperceived social norms among high-risk college student groups. Addictive Behaviors 35: 1094-101. [CrossRef] [PubMed]

Leigh, Barbara C., and Alan W. Stacy. 1993. Alcohol outcome expectancies: Scale construction and predictive utility in higher order confirmatory models. Psychological Assessment 5: 216. [CrossRef]

Lewis, Melissa A., and Clayton Neighbors. 2004. Gender-specific misperceptions of college student drinking norms. Psychology of Addictive Behaviors 18: 334. [CrossRef]

Licciardone, John C. 2003. Perceptions of drinking and related findings from the nationwide campuses study. Journal of American College Health 51: 238-45. [CrossRef] [PubMed]

Lin, Carolyn A., Jamie Harris, and Carolyn Lagoe. 2014. Formative research on identifying and promoting responsible party-hosting skills among college students. Journal of Communication in Healthcare 7: 272-84. [CrossRef]

Lin, Carolyn A., and Jeffrey R. Carlson. 2009. Theorizing college students' high-risk drinking behavior: An exploratory study. In Conference Papers. International Communication Association, Annual Meeting, 1-32.

Lovecchio, Catherine P., Todd M. Wyatt, and William DeJong. 2010. Reductions in drinking and alcohol-related harms reported by first-year college students taking an online alcohol education course: A randomized trial. Journal of Health Communication 15: 805-19. [CrossRef] [PubMed]

McAlaney, John, and John McMahon. 2007. Normative beliefs, misperceptions, and heavy episodic drinking in a British student sample. Journal of Studies on Alcohol and Drugs 68: 385-92. [CrossRef]

Merrill, Jennifer E., Holly K. Boyle, Nancy P. Barnett, and Kate B. Carey. 2018. Delivering normative feedback to heavy drinking college students via text messaging: A pilot feasibility study. Addictive behaviors 83: 175-81. [CrossRef]

Miller, Mary Beth, Eliza Van Reen, David H. Barker, Brandy M. Roane, Brian Borsari, John E. McGeary, Ronald Seifer, and Mary A. Carskadon. 2017. The impact of sleep and psychiatric symptoms on alcohol consequences among young adults. Addictive Behaviors 66: 138-44. [CrossRef] 
Montano, Daniel E., and Danuta Kasprzyk. 2015. Theory of reasoned action, theory of planned behavior, and the integrated behavioral model. Health Behavior: Theory, Research and Practice 70: 231.

Mullen, Brian, and Jerry Suls. 1982. "Know thyself": Stressful life changes and the ameliorative effect of private self-consciousness. Journal of Experimental Social Psychology 18: 43-55. [CrossRef]

National Institute on Alcohol Abuse and Alcoholism (NIAAA). 2021. College Drinking Fact Sheet. Available online: https://www. collegedrinkingprevention.gov/NIAAACollegeMaterials/FactSheets/collegedrinkingfactsheet.aspx (accessed on 1 July 2021).

NIAAA National Advisory Council. 2004. NIAAA Council Approves Definition of Binge Drinking. NIAAA Newsletter 3. Available online: https:/ / pubs.niaaa.nih.gov/publications/Newsletter/winter2004/Newsletter_Number3.pdf (accessed on 1 July 2021).

Norman, Paul. 2011. The theory of planned behavior and binge drinking among undergraduate students: Assessing the impact of habit strength. Addictive Behaviors 36: 502-7. [CrossRef]

Norman, Paul, Christopher J. Armitage, and Catherine Quigley. 2007. The theory of planned behavior and binge drinking: Assessing the impact of binge drinker prototypes. Addictive Behaviors 32: 1753-68. [CrossRef] [PubMed]

Norman, Paul, and Sarah Hoyle. 2004. The theory of planned behavior and breast self-examination: Distinguishing between perceived control and self-efficacy. Journal of Applied Social Psychology 34: 694-708. [CrossRef]

Pan, Yanfang, and Peida Zhan. 2020. The Impact of Sample Attrition on Longitudinal Learning Diagnosis: A Prolog. Frontiers in Psychology 11: 1051. [CrossRef] [PubMed]

Papachristou, Harilaos, Evi Aresti, Marios Theodorou, and Georgia Panaylotou. 2018. Alcohol outcome expectancies mediate the relationship between social anxiety and alcohol drinking in university students: The role of gender. Cognitive Therapy and Research 42: 289-301. [CrossRef]

Paschall, Mallie J., Chris Ringwalt, Todd Wyatt, and William DeJong. 2014. Effects of an online alcohol education course among college freshmen: An investigation of potential mediators. Journal of Health Communication 19: 392-412. [CrossRef]

Paschall, Mallie J., Melina Bersamin, Melodie Fearnow-Kenney, David Wyrick, and David Currey. 2006. Short-term evaluation of a web-based college alcohol misuse and harm prevention course (College Alc). Journal of Alcohol and Drug Education 50: 49.

Pasupathi, Monisha. 1999. Age differences in response to conformity pressure for emotional and nonemotional material. Psychology and Aging 14: 170. [CrossRef]

Perkins, H. Wesley, and David W. Craig. 2006. A successful social norms campaign to reduce alcohol misuse among college student-athletes. Journal of Studies on Alcohol 67: 880-89. [CrossRef]

Rimal, Rajiv N., and Kevin Real. 2005. How behaviors are influenced by perceived norms: A test of the theory of normative social behavior. Communication Research 32: 389-414. [CrossRef]

Rivis, Amanda, and Paschal Sheeran. 2003. Descriptive norms as an additional predictor in the theory of planned behaviour: A meta-analysis. Current Psychology 22: 218-33. [CrossRef]

Scott-Sheldon, Lori AJ, Kate B. Carey, Tyler S. Kaiser, Jennifer M. Knight, and Michael P. Carey. 2016. Alcohol interventions for Greek letter organizations: A systematic review and meta-analysis, 1987 to 2014. Health Psychology 35: 670. [CrossRef] [PubMed]

Substance Abuse and Mental Health Services Administration (SAMHSA). 2019. National Survey on Drug Use and Health (NSDUH). Table 6.21B-Types of Illicit Drug, Tobacco Product, and Alcohol Use in Past Month among Persons Aged 18 to 22, by College Enrollment Status and Gender: Percentages, 2018 and 2019. Available online: https://www.samhsa.gov/data/sites/default/files/reports/rpt29394 /NSDUHDetailedTabs2019/NSDUHDetTabsSect6pe2019.htm\#tab6-21b (accessed on 1 July 2021).

Thombs, Dennis L., R. Scott Olds, Cynthia J. Osborn, Sarah Casseday, Kevin Glavin, and Alan D. Berkowitz. 2007. Outcomes of a technology-based social norms intervention to deter alcohol use in freshman residence halls. Journal of American College Health 55 : 325-32. [CrossRef]

Trafimow, David. 1996. The importance of attitudes in the prediction of college students' intentions to drink. Journal of Applied Social Psychology 26: 2167-88. [CrossRef]

Van der Pligt, Joop, and J. Richard Eiser. 1984. Dimensional salience, judgment, and attitudes. In Attitudinal Judgment. New York: Springer, pp. 161-77.

Vergés, Alvaro, Kristina M. Jackson, Kathleen K. Bucholz, Julia D. Grant, Timothy J. Trull, Phillip K. Wood, and Kenneth J. Sher. 2012. Deconstructing the age-prevalence curve of alcohol dependence: Why "maturing out" is only a small piece of the puzzle. Journal of Abnormal Psychology 121: 511. [CrossRef]

Willis, Loren, Eunro Lee, Katherine J. Reynolds, and Kathleen A. Klik. 2020. The theory of planned behavior and the social identity approach: A new look at group processes and social norms in the context of student binge drinking. Europe's Journal of Psychology 16: 357-83. [CrossRef]

Wechsler, Henry, Jae Eun Lee, Meichun Kuo, and Hang Lee. 2000. College binge drinking in the 1990s: A continuing problem results of the Harvard School of Public Health 1999 College Alcohol Study. Journal of American College Health 48: 199-210. [CrossRef]

Zucker, Robert A. 1994. Pathways to alcohol problems and alcoholism: A developmental account of the evidence for multiple alcoholisms and for contextual contributions to risk. The Development of Alcohol Problems: Exploring the Biopsychosocial Matrix of Risk 26: 255-89. 